\title{
Original
}

\section{Medication Replacing Conventional with Atypical Antipsychotic : Effect on Cognitive Function in Schizophrenic Patients}

\author{
Takafumi InADA, Masaru Mimura, \\ Takahiro KATo and Kunitoshi KamiJima
}

\begin{abstract}
We studied whether or not replacing conventional antipsychotic medications with newer atypical antipsychotics has a favorable effect on cognitive function in schizophrenia. Twenty patients with schizophrenia who had been treated with conventional antipsychotics were switched randomly to either of two atypical antipsychotic agents, risperidone or perospirone. Clinical and neuropsychological evaluations were performed before and approximately four weeks after completing medication change. Patients required a lower dose of antiparkinsonian drugs and presented fewer parkinsonian symptoms while taking atypical antipsychotics compared with conventional antipsychotics. In addition, patients taking perospirone required smaller antiparkinsonian drug dose than those taking risperidone, suggesting that perospirone caused fewer extrapyramidal adverse effects. Perospirone roughly paralleled risperidone in diminishing psychotic symptoms but these were fewer and the subjectively assessed quality of life was better in patients treated with risperidone. Improvement in neuropsychological test results upon switching to atypical agents was remarkable for verbal memory and various measures of frontal/executive functions. The effect of risperidone and perospirone on frontal/executive functions appeared equivalent while changing to risperidone rather than perospirone achieved greater improvement in verbal memory. Differences in verbal memory favoring risperidone may reflect a favorable balance between D2 dopamine and 5HT2A serotonin receptor blockade. Further study is needed concerning how this cognitive improvement might be related to improved social functioning.
\end{abstract}

Key words : schizophrenia, atypical antipsychotics, cognitive function, risperidone, perospirone

\section{Introduction}

Schizophrenia presents with characteristic neurocognitive deficits in addition to the well-known positive and negative symptoms. In fact, social prognosis for schizophrenic patients has been reported to be more closely related to these impairments than to positive and negative symptoms ${ }^{1-3)}$. Improving cognitive function therefore should be a key goal in

Department of Neuropsychiatry, Showa University School of Medicine, 1-5-8 Hatanodai, Shinagawa-ku, Tokyo 1428666, Japan. 
treatment. However, typical antipsychotic agents may have little effect or even a negative impact on cognitive functions ${ }^{4,5)}$. Frequent use of concomitant anticholinergic antiparkinsonian drugs may further compromise cognitive functions including short-term memory ${ }^{6,7}$. Recent studies have demonstrated that use of newer atypical antipsychotics can improve neurocognitive function $^{8-10)}$.

Beneficial properties of atypical antipsychotics compared to conventional agents include improved effectiveness against negative symptoms with fewer adverse effects such as extrapyramidal syndromes (EPS) and hyperprolactinemia. In addition, improvement of cognitive functions brought about by atypical agents may enhance social prognosis in schizophrenia ${ }^{11}$. The principal aim of the present study was to determine whether or not replacing conventional with atypical agents has a measurable favorable impact on cognitive functions in patients with schizophrenia.

Four atypical antipsychotic agents are currently available in Japan: risperidone, olanzapine, quetiapine, and perospirone. We specifically focused on risperidone and perospirone in the present study. Perospirone was developed in Japan, where it was approved for clinical use only three years ago. This drug has not yet been approved in Western countries and therefore information concerning this agent is limited. In particular, we have little knowledge of the concerning cognitive effects of perospirone in schizophrenia. Olanzapine and quetiapine are not recommended for patients with hyperglycemia, hyperlipidemia, or obesity, which limits our ability to study these agents in a randomized clinical trial. We therefore chose risperidone and perospirone for this study. We randomly switched treatment regimens of schizophrenic patients from conventional agents to either risperidone or perospirone, while assessing cognitive changes using a detailed neuropsychological test battery.

\section{Methods}

\section{Subjects and change of medication}

Subjects included 20 schizophrenic outpatients or inpatients at Showa University Hospital ( 9 males, 11 females). Candidates whose age exceeded 65 years were excluded in order to avoid age-related effects. Psychiatric diagnosis was based on items F20 to 29 according to the criteria of the International Classification of Disease, 10th edition (ICD-10). Overall severity was evaluated using the Clinical Global Impression (CGI) ${ }^{12}$. Positive and negative symptoms in each patient were evaluated together with the Brief Psychiatric Rating Scale $(\text { BPRS })^{13)}$. EPS were rated according to the Drug Induced Extrapyramidal Symptoms Scale (DIEPSS $)^{14)}$. The EuroQOL ${ }^{15)}$ was used as an index for the subjective quality of life (QOL). At the time of entry all patients were being treated with conventional antipsychotic agents including haloperidol, chlorpromazine, bromperidol and thioridazine. Eighty-five percent of patients $(17 / 20)$ were also taking anticholinergic antiparkinsonian agents (biperiden and trihexyphenidyl). To facilitate comparison, the total dose of antipsychotic medication was converted to chlorpromazine equivalents, while the total dose of antiparkinsonian drugs was converted to biperiden equivalents ${ }^{16,17}$.

Each patient was randomly assigned to receive either risperidone or perospirone, and all patients' conventional antipsychotic agents were replaced accordingly. Details of tapering the dose of the conventional drug and increasing the dose of the atypical drug depended upon each patient's clinical condition, but all patients completed the change of medication 
Table 1. Demographic and baseline clinical data for randomly determined treatment group

\begin{tabular}{llll}
\hline \multicolumn{1}{c}{ Group } & Risperidone & & Perospirone \\
\hline $\mathrm{N}$ & 10 & 10 \\
Genders, M/F & $3 / 7$ & $6 / 4$ \\
Age, years* & $52.7(9.2)$ & & $45.4(10.7)$ \\
\hline
\end{tabular}

${ }^{*}$ Data are presented as the mean $(\mathrm{SD})$.

within two months. The mean dose of atypical antipsychotic was $3.7 \mathrm{mg}$ (range, 1.5 to 6.0 ) in the risperidone group and $20.0 \mathrm{mg}$ (range, 8.0 to 40.0 ) in the perospirone group. Table 1 presents patients' demographic and baseline clinical data according to group. No difference was evident between risperidone and perospirone groups with regard to age, duration of illness, BPRS and DIEPSS scores.

\section{Evaluation of clinical symptoms and cognitive function}

Clinical evaluation using the CGI, BPRS, DIEPSS, and EuroQOL was repeated approximately four weeks after completion of the change of antipsychotic medication. The following neuropsychological test battery was administered before and four weeks after the change to investigate neurocognitive function in various domains. Neuropsychological tests were administered by a psychologist who was unaware of medication regimens. The test battery included 11 components: reaction time, digit span, block design, symbol digit modalities, figure position, the Rey Auditory Verbal Learning Test (RAVLT) ${ }^{18)}$, trailmaking, the Stroop test, word fluency, a key search, and the zoo map. The last two components are subtests of the Behavioral Assessment of the Dysexecutive Syndrome (BADS $)^{19,20)}$.

Reaction time, block design, symbol digit modalities, figure position, and trail-making were tested using a computer-administered test battery (Neuropsychological Tests, CDROM) devised by the Japan Society of Hepatology. Both the instructions and stimuli were presented by computer, and subject responses were made on a touch-panel monitor. Brief descriptions of each test are given below, while detailed descriptions can be found in Moriyama et $a l^{21)}$.

\section{Reaction time}

The subject was asked to press a button as soon as a red circle appeared on the touch-panel monitor. The test included three versions: A, consecutive brief presentation of red circles only; B, random presentation of red, yellow, and blue circles (task: press the button when a red circle appeared); and $\mathrm{C}$, random presentation of red, yellow, and blue circles (task: press the button only when a red circle is followed by a yellow circle). Each version lasted for $60 \mathrm{~s}$, and the mean time required for correct reactions was computed. Reaction times obtained by this test may reflect information processing speed.

\section{Block design}

This task was nearly identical to the WAIS-R subtest except that it was presented by computer. The subject was asked to copy a model made up of four red/white cards. The 
number of correct responses (maximum 5, compared with 10 in the original version), and the summed time required for completion was computed. This task was intended to assess visuoconstructional ability.

\section{Symbol digit modalities}

The subject was asked to match a symbol to a digit. The number of correct responses achieved within 60 s was calculated (compared with $90 \mathrm{~s}$ in the original version). While this task can be interpreted in several ways, it may reflect visual-motor processing and ability to divide one's attention.

\section{Figure position}

The subject was asked to memorize two to four figures (e.g. a triangle and a square) together with their positions among six possible arrangements. The subject was then asked to recall the figures and their positions after $15 \mathrm{~s}$ intervals. The total number of correct responses was recorded (maximum, 9). This task was used to evaluate nonverbal short-term memory.

\section{Rey auditory verbal learning test $(R A V L T)^{18)}$}

Verbal memory was assessed using the RAVLT, in which the subject was asked to memorize a spoken list of 15 common nouns. The maximum number of words recalled correctly in any order within five consecutive trials was used for analysis.

Executive function was assessed using the following five instruments: trail-making tests, the Stroop test, word fluency, a key search test, and the zoo map test.

\section{Trail-making $A$}

The subject was asked to draw lines to connect 25 consecutively numbered circles distributed randomly on the screen. Time for completion was recorded. The trail-making A tests may reflect visuomotor and attentional processing.

\section{Trail-making $B$}

The subject was asked to draw lines to connect 25 consecutively numbered and lettered circles by alternating between the two sequences. Time for completion was recorded. Like trail-making $\mathrm{A}$, this test concerns visuomotor and attentional processing, but it includes the additional element of alternating or dividing attention.

\section{Stroop test}

The subject was asked to name the color of randomly arranged colored and labeled dots (red, green, yellow, and blue) in a congruent condition (version A), a neutral condition (version B), and an incongruent condition (version C). Time for completion was measured, and the time difference between versions $\mathrm{A}$ and $\mathrm{C}$ (C-A) was used as a variable for analysis.

\footnotetext{
Word fluency

Within one minute, the subject was asked to name as many nouns as possible that began with a given initial letter (initial word fluency) or those that belonged to a specific
} 
semantic category (category word fluency).

Key search

The subject was given a piece of paper with a $100-\mathrm{mm}$ square drawn on it and asked to imagine that the square was a large field in which he/she somewhere had lost a key. The subject was instructed to draw a line to show how to search the field in order to find the lost key. This subtest examines ability to solve an open-ended problem.

\section{Zoo map}

The subject was asked to map out a route to visit predetermined places in a zoo according to a designated rule. In the first part, the subject had to plan the order in which locations were visited. In the second part, this order was specified by the examiner as an aid in the solving problem. This subtest examines the abilities to plan and to use feedback in problem solving.

\section{Statistical analyses}

Doses of antipsychotic and antiparkinsonian drugs were subjected to analysis of variance (ANOVA). Each clinical and cognitive measure was also subjected to ANOVA to compare the effect of each drug. ANOVAs were conducted with one "within" factor (time: before vs. after the drug change) and one "between" factor (drug: risperidone vs. perospirone). Additional individual ANOVAs were conducted to assess the risperidone and perospirone groups separately. Bonferroni's Post-hoc tests were introduced when needed. Statistical significance was set at $\mathrm{p}<.05$.

\section{Ethical issues}

Oral informed consent was obtained from each patient before beginning the baseline tests. The study was approved by the Ethics Committee of Showa University.

\section{Results}

\section{Doses of antipsychotic and antiparkinsonian agents}

Doses of antipsychotic and antiparkinsonian agents after the drug change are shown in Table 2. For antipsychotic agent dose, the main effect of atypical antipsychotic drug group was not significant $[F(1,18)=0.004, p>.10]$ but the main effect of time was significant $[F(1,18)=4.29, p<.05]$, indicating that patients required lower antipsychotic drug doses after changing medication. Interestingly, the drug $x$ time interaction was significant $[F(1,18)=5.66, p<.05]$, which suggested that changing to perospirone permitted a larger decrease in dose than changing to risperidone (Bonferroni's post-hoc test, $\mathrm{p}<.05$ for perospirone, $\mathrm{p}>.10$ for risperidone).

Similarly, for antiparkinsonian agent dose, the main effect of atypical antipsychotic drug group was not significant $[F(1,18)=0.004, p>.10]$ while the main effect of time was highly significant $[F(1,18)=24.75, p<.0001]$, indicating that patients required much lower doses of antiparkinsonian agents after completing the change in medication. The drug $x$ time interaction showed a trend toward significance $[F(1,18)=2.75, p=.10]$, suggesting that perospirone required lower doses of antiparkinsonian agents than risperidone (Bonferroni's post-hoc test, $\mathrm{p}<.01$ for perospirone, $\mathrm{p}=.03$ for risperidone). 
Table 2. Clinical variables and drug dose equivalents before and after changing medication

\begin{tabular}{lcrrr}
\hline \multirow{2}{*}{ Group } & \multicolumn{2}{c}{ Risperidone } & \multicolumn{2}{c}{ Perospirone } \\
\cline { 2 - 5 } & \multicolumn{1}{c}{ pre } & \multicolumn{1}{c}{ post } & pre & post \\
\hline CGI & $3.7(0.9)$ & $3.3(0.8)$ & $3.5(0.8)$ & $3.3(0.5)$ \\
BPRS & $40.1(9.4)$ & $35.4(5.9)^{*}$ & $42.0(16.9)$ & $39.8(14.0)$ \\
DIEPSS & $1.4(0.7)$ & $1.2(0.4)$ & $1.7(0.2)$ & $1.2(0.4)$ \\
EuroQOL & $7.6(1.6)$ & $6.9(1.7)^{*}$ & $7.2(2.3)$ & $7.5(3.0)$ \\
Antipsychotic dose & $430.0(264.3)$ & $446.7(261.9)$ & $551.4(347.6)$ & $311.1(194.9)^{*}$ \\
Antiparkinsonian dose & $3.5(2.8)$ & $2.3(2.4)^{*}$ & $4.1(3.5)$ & $1.6(3.4)^{* * *}$ \\
\hline
\end{tabular}

Data are presented as the mean (SD). Dose of antipsychotic and antiparkinsonian agents are shown as chlorpromazine and biperiden equivalents, respectively.

BPRS, Brief Psychiatric Rating Scale; CGI, Clinical Global Impression; DIEPSS, Drug Induced Extrapyramidal Symptoms Scale, QOL, Quality of Life.

${ }^{*} \mathrm{p}<.05,{ }^{* *} \mathrm{p}<.01,{ }^{* * *} \mathrm{p}<.001$ for data after changing vs. those obtained before.

\section{Changes in clinical scores}

Changes in CGI, BPRS, DIEPSS, and EuroQOL are shown in Table 2. The CGI was not significantly changed by the switch in treatment. However, the ANOVA for BPRS yielded a significant main effect of time $[F(1,18)=5.30, p=.03]$, while neither the main effect of the atypical antipsychotic drug group $[F(1,18)=0.32, p>.10]$ nor the time $x$ drug interaction $[F(1,18)=0.72, p>.10]$ was significant. Thus both risperidone and perospirone were more effective in ameliorating psychotic symptoms than conventional drugs. Additional individual ANOVAs showed that improvement of the BPRS was significant for risperidone (Bonferroni's post-hoc test, $\mathrm{p}<.05$ ), while a trend towards significance was obtained for perospirone (Bonferroni's post-hoc test, $0.5<\mathrm{p}<1.0$ ).

Improvement of the DIEPSS was comparable for risperidone and perospirone, with both generally being superior to conventional drugs [main effect of time, $F(1,18)=5.04, p=.04$; main effect of drug group, $F(1,18)=0.12, p>.10$ ]. The time $\mathrm{x}$ drug interaction was not significant $[F(1,18)=0.72, p>.10]$. Additional individual ANOVAs for risperidone and perospirone did not demonstrate significance.

As for the EuroQOL, the main effect of time was not significant $[F(1,18)=1.00, p>.10]$. The main effect of the atypical antipsychotic drug group $[F(1,18)=3.48, p=.08]$ as well as the time $\mathrm{x}$ drug interaction $[\mathrm{F}(1,18)=3.48, \mathrm{p}=.08]$ approached significance. Additional individual ANOVAs indicated that improvement was significant only for risperidone (Bonferroni's post-hoc test, $\mathrm{p}<.05$ ).

\section{Change in cognitive measures}

Results of neuropsychological testing before and after the drug change are shown in Table 3. Significant improvement was observed in domains including verbal memory and executive functions. The main effect of time was significant for the RAVLT $[F(1,18)=8.22$, $\mathrm{p}<.01]$, symbol digit modalities $[\mathrm{F}(1,18)=10.73, \mathrm{p}<.01]$, trail-making $\mathrm{B}[\mathrm{F}(1,18)=3.90$, $\mathrm{p}<.05]$, initial word fluency $[\mathrm{F}(1,18)=4.48, \mathrm{p}<.05]$, and category word fluency $[\mathrm{F}(1,18)=5.53, \mathrm{p}<.05]$. Differences in visual memory and stepwise problem solving (zoo map) approached significance [figure position, $F(1,18)=2.88, p=.10 ;$ zoo $\operatorname{map}, F(1,18)=$ 
Table 3. Neuropsychological test results before and after changing medication

\begin{tabular}{lcrcr}
\hline \multicolumn{1}{c}{ Group } & \multicolumn{2}{c}{ Risperidone } & \multicolumn{2}{c}{ Perospirone } \\
\cline { 2 - 5 } & \multicolumn{1}{c}{ pre } & \multicolumn{1}{c}{ post } & pre & post \\
\hline Reaction time A, sec & $0.44(0.11)$ & $0.45(0.07)$ & $0.49(0.14)$ & $0.54(0.17)$ \\
Reaction time B, sec & $0.46(0.07)$ & $0.45(0.09)$ & $0.46(0.07)$ & $0.46(0.10)$ \\
Reaction time C, sec & $0.47(0.10)$ & $0.44(0.08)$ & $0.42(0.03)$ & $0.43(0.04)$ \\
Block design & $4.4(0.7)$ & $4.7(0.7)$ & $4.8(0.5)$ & $4.8(0.5)$ \\
Symbol digit & $11.7(5.9)$ & $16.3(4.7)^{*}$ & $13.8(5.9)$ & $18.1(5.3)^{*}$ \\
Figure position & $5.9(2.2)$ & $7.1(1.4)$ & $7.4(1.3)$ & $7.9(1.6)$ \\
RAVLT, maximum & $8.1(1.1)$ & $10.8(2.8)^{* *}$ & $11.0(2.8)$ & $11.2(2.8)$ \\
Trail-making A, sec & $53.6(22.4)$ & $48.2(22.9)$ & $48.2(20.4)$ & $40.7(15.0)$ \\
Trail-making B, sec & $122.1(45.5)$ & $101.8(51.0)^{*}$ & $96.5(46.8)$ & $71.1(36.6)^{*}$ \\
Stroop C-A, sec & $9.0(8.9)$ & $11.3(3.7)$ & $14.9(8.9)$ & $10.9(5.8)$ \\
Word fluency, initial & $7.8(3.3)$ & $9.4(4.2)^{*}$ & $7.3(2.1)$ & $8.5(2.9)^{*}$ \\
category & $12.4(2.5)$ & $14.4(2.5)^{*}$ & $11.6(3.2)$ & $12.8(3.3)^{*}$ \\
Key search & $2.0(1.2)$ & $2.8(0.9)$ & $2.2(1.2)$ & $2.2(1.0)$ \\
Zoo map & $12.3(3.8)$ & $13.0(3.9)$ & $9.3(4.4)$ & $12.4(3.4)$ \\
\hline
\end{tabular}

Data are presented as the mean (SD).

${ }^{*} \mathrm{p}<.05,{ }^{* *} \mathrm{p}<.01$ for date after changing vs. those obtained before.

RAVLT, Rey Auditory Verbal Learning Test.

3.12, $\mathrm{p}=.09]$. The main effect of the atypical antipsychotic drug group was not significant for the most of the measures listed above, suggesting an overall equal level of performance before switching treatment. Similarly, the drug $\mathrm{x}$ time interaction did not show significance, indicating that improvement with risperidone paralleled that with perospirone. The only exception to this generalization involved verbal memory, where patients taking risperidone demonstrated significantly greater improvement than those taking perospirone $[F(1,18)=5.91, p=.02]$. Additional individual ANOVAs indicated that improvement in the RAVLT was significant only for risperidone (Bonferroni's post-hoc test, $\mathrm{p}<.05$ ).

The main effect of time was not significant for reaction time $A[F(1,18)=0.79]$, reaction time $\mathrm{B}[\mathrm{F}(1,18)=0.01]$, reaction time $\mathrm{C}[\mathrm{F}(1,18)=0.11]$, block design $[\mathrm{F}(1,18)=0.27]$, trail-making A $[F(1,18)=1.40]$, Stroop C-A $[F(1,18)=0.17]$, or the key search $[F(1,18)=2.47]($ all $p>.10)$. Neither the main effect of the atypical antipsychotic drug group nor the drug $\mathrm{x}$ time interaction was significant for these scores.

\section{Discussion}

\section{Favorable effect of atypical antipsychotics on parkinsonian symptoms}

As shown by the results for the DIEPSS, both risperidone and perospirone had fewer parkinsonian adverse effects than conventional antipsychotics. Consequently, both drugs required lower doses of antiparkinsonian agents. Moreover, the decrease in dose of antiparkinsonian agents was larger in the perospirone group than in the risperidone group. These results confirmed previous reports that atypical antipsychotics, especially perospirone, caused fewer parkinsonian adverse effects ${ }^{22}$. Perospirone thus appeared particularly suitable for patients with prominent parkinsonian symptoms. A pharmacologic profile of perospirone suggests a smaller association with EPS than is noted for risperidone ${ }^{22}$. 
Atypical antipsychotics and change in clinical symptoms

Both atypical antipsychotic agents similarly decreased psychotic symptoms compared with symptoms persisting during treatment with conventional drugs. Risperidone may be more able than perospirone to decrease psychotic symptoms and enhance subjectively assessed QOL, as indicated by respective changes in BPRS and EuroQOL. However, since we adopted a flexible-dose protocol and the average dose of perospirone appeared to be less than that of risperidone when expressed in equivalent units, we cannot draw a strong conclusion. Our limited clinical experience with perospirone may have contributed to the relatively low doses prescribed for this new agent. Higher doses of perospirone might ultimately achieve better alleviation of psychotic symptoms.

The effect on clinical features of changing from conventional to atypical agents was relatively small. This may reflect the specific study population, including mostly outpatients in a chronic stage, whose symptoms had been at least partially controlled by conventional agents. A second factor that might have limited the effect of switching medications was the short duration of treatment with the atypical agent before clinical reevaluation: approximately one month after completing the change. Sufficient length of observation is important for evaluating psychotic symptoms and subjective QOL. While atypical antipsychotics including risperidone and olanzapine have been reported to improve subjective QOL $^{23)}$, Montes and colleagues $^{23)}$ reported significant differences in the EuroQOL in first-episode schizophrenia between conventional and atypical antipsychotic drugs after six months of treatment. Accordingly, long-term follow-up in our study population may reveal more robust clinical differences between conventional and atypical agents.

\section{Atypical antipsychotics and changes in cognitive functions}

Both risperidone and perospirone were significantly effective in cognitive domains including verbal memory and executive functions. In contrast, switching to one of these had a considerably smaller effect on visuoconstructive ability, visual memory, and simple attention. These results are consistent with previous findings concerning atypical antipsychotic drugs and cognitive function in schizophrenia ${ }^{24)}$. Previous studies suggested that specific atypical agents may have differential effects on individual cognitive domains ${ }^{24)}$. Risperidone has been reported to have a stronger positive effect on working memory and verbal function than other atypical agents ${ }^{8)}$. Our results partially confirmed this favorable effect of risperidone on verbal memory. In considering prognosis in schizophrenia, improvement of verbal memory by atypical agents, specifically risperidone, could be of great importance. Green and colleagues ${ }^{2,3)}$ emphasized that verbal memory is intimately related to prognosis with respect to social functioning in schizophrenic patients. Appropriate pharmacologic treatment as well as psychosocial intervention could optimize functional recovery in schizophrenia.

Negative and disorganized symptoms, as opposed to positive symptoms, have been linked with cognitive dysfunctions ${ }^{25)}$. Since the present analysis did not include ratings that evaluated positive and negative symptom domains separately, we could not address this issue. However, we could argue more generally that neuropsychological indices were sensitive measures likely to demonstrate changes associated with a medication switch after approximately two to three months.

The impact profile of perospirone on cognitive function in schizophrenia was almost 
identical to that of risperidone. Since perospirone was developed only recently in Japan and is currently not approved for clinical use in Western countries, information concerning the drug still is still incomplete. As far as we know, this is the first study to describe the effect of perospirone on cognitive functions as determined by a detailed neuropsychological test battery. Perospirone blocks dopamine receptors and blocks 5HT2 serotonin receptors even more strongly, thus acting as a serotonin-dopamine antagonist (SDA) similar to risperidone. One unique characteristic of perospirone is that it demonstrates a stronger action as a 5HT1A receptor agonist than is found with risperidone ${ }^{26}$.

Studies have found that 5HT1A agonists increase dopamine release in the prefrontal cortex and enhance cognitive functions ${ }^{27,28)}$. Sumiyoshi et al. ${ }^{29)}$ reported that verbal memory in patients with schizophrenia may benefit from tandospirone, a partial agonist at 5HT1A receptors. Accordingly, we initially hypothesized that perospirone, a 5HT1A agonist, would improve verbal memory more than risperidone. However, we found that both atypical agents enhanced verbal memory, and in fact improvement of verbal memory was greater with risperidone. One alternative hypothesis might be that the ratio of D2 dopamine to 5HT2A serotonin blockade determines a drug's potential for enhancing verbal memory. An in vivo experiment demonstrated that this D2/5-HT2A antagonism ratio was 20.6 for risperidone and 2.3 for perospirone ${ }^{30)}$. It is therefore reasonable to suggest that balance between D2 and 5HT2A blockade may influence verbal memory. Further studies are warranted to investigate possible dissociable mechanisms of action of the drugs presently studied concerning specific cognitive functions.

\section{Limitations and clinical implications of this study}

Several possible weaknesses in the present study should be acknowledged. Firstly, although the trial was randomized and prospective, no double blind was maintained concerning the drug given. However, the psychologist who examined neuropsychological functions was kept uninformed of medication status. Accordingly, neuropsychological results were probably reasonably free from examiner' bias. Secondly, the number of subjects was relatively small, 10 per group and thirdly, the short study duration may have been problematic. These factors could weaken statistical power. Further studies with a larger number of patients and longer duration of treatment are warranted. A fourth problem is an antipsychotic dose bias. For the perospirone group, the mean conventional antipsychotic dose before starting this study was greater than that for risperidone group. In addition, the mean dose after switching medications was much smaller for the perospirone group. Thus, there might be a bias in comparing the effects of perospirone and risperidone. Another problem could be a possible repetition effect. Subjects may have improved their performance at the administration of the second test simply from repeating the same tasks performed previously. As reported in a previous study, however, repetition effect with the neuropsychological tests included here should be negligible ${ }^{21}$. To more completely avoid this problem, future studies should include two-step baseline testing. An alternative solution is to include a set of control patients repeating neuropsychological tests without changing medications.

Despite the limitations above, the present study has important clinical implications. Besides a mild favorable effect on EPS and psychotic symptoms, changing to atypical antipsychotic agents led to a fairly robust improvement in cognitive functioning. Most 
significant improvements were observed in verbal memory and frontal/executive domains. While risperidone and perospirone appeared to be nearly identical in ameliorating frontal/ executive dysfunction, the former was superior to the latter in terms of verbal memory and subjective QOL. The reverse was true for minimizing EPS. These findings should guide medication choices in clinical practice. Future studies are needed to more directly clarify how ameliorating cognitive dysfunction by using atypical antipsychotics may improve psychosocial and interpersonal functioning in schizophrenia.

\section{References}

1) Addington $\mathrm{J}$ and Addington $\mathrm{D}$ : Neurocognitive and social functioning in schizophrenia: a 2.5 year follow-up study. Schizophr Res $44:$ 47-56 (2000)

2) Green MF : What are the functional consequences of neurocognitive deficits in schizophrenia? Am J Psychiatry 153 : 321-330 (1996)

3) Green MF and Nuechterlein KH : Should schizophrenia be treated as a neurocognitive disorder? Schizophr Bull 25 : 309-318 (2000)

4) Melzer HM, Casey DE and Garver DL : Evaluating the effects of antipsychotics on cognition in schizophrenia. J Clin Psychiatry 59(Suppl 12) : 35-40 (1998)

5) Stip E: Memory impairment in schizophrenia: perspectives from psychopathology and pharmacology. Can $J$ Psychiatry $41: 27-34$ (1996)

6) Fayen M, Goldman MB, Moulthrop MA and Luchins DJ : Differential memory function with dopaminergic versus anticholinergic treatment of drug-induced extrapyramidal symptoms. Am J Psychiatry 145: 483-486 (1988)

7) Heinik J : Effects of trihexyphenidyl on MMSE and CAMCOG scores of medicated elderly patients with schizophrenia. Int Psychogeriatr 10 : 103-108 (1998)

8 ) Cuesta MJ, Peralta V and Zarzuela A : Effects of olanzapine and other antipsychotics on cognitive function in chronic schizophrenia : a longitudinal study. Schizophr Res 48 : 17-28 (2001)

9) Harvey PD, Moriaty PJ, Serper MR, Schnur E and Lieber D: Practice-related improvement in information processing with novel antipsychotic treatment. Schizophr Res $46: 139-148$ (2000)

10) Kern RS, Green MF, Marshall BD Jr, Wirshing WC, Wirshing D, McGurk SR, Marder SR and Mintz J : Risperidone versus haloperidol on secondary memory : can newer medications aid learning? Schizophr Bull 25 : 223-232 (1999)

11) Lindstrom LH : Long-term clinical and social outcome studies in schizophrenia in reaction to the cognitive side effects of antipsychotic drugs. Acta Psychiatr Scand 380(Suppl) : 74-76 (1994)

12) National Institute of Mental Health: Clinical Global Impression (CGI). In : ECDUE Assessment manual for psychopharmacology (Revised) Guy W (Ed), U.S. Department of Health, Education, and Welfare, Public Health Service, Rockville, pp 217-222 (1976)

13) Kolakowska T: Brief psychiatric rating scale : glossary and rating instructions. Oxford University Press, Oxford (1976)

14) Inada $T$ and Yagi $G$ : Current topics in neuroleptic-induced extrapramidal symptoms in Japan. Keio $J$ Med 45 : 95-99 (1996)

15) EuroQol Group: EuroQol : a new facility for the measurement of health-related quality of life. Health Policy 16 : 199-208 (1990)

16) Inagaki A, Inada T, Fujii Y and Yagi G : Dose equivalence of psychotropic drugs. part XV : Dose equivalence of novel neuroleptics. Jpn J Clin Psychopharmacol 4 : 869-870 (2001)

17) Inagaki A, Inada $T$, Fujii $Y$, Yagi G, Yosio $T$, Nakamura $H$ and Yamauchi $T$ : Dose equivalence of antiparkisonian drugs. In : Dose equivalence of psychotropic drugs. Seiwa Press, Tokyo, pp 61-76 (1999)

18) Lezak M: Neuropsychological Assessment, $3^{\text {rd }}$ edition, Oxford University Press, New York (1995)

19) Wilson BA, Alderman N, Burgess PW, Emslie H and Evans JJ : Behavioural Assessment of the Dysexecutive Syndrome. Thames Vally Test Company, Bury, St. Edmunds (1996)

20) Evans JJ, Chua SE, Mckenna PJ and Wilson BA: Assessment of the dysexecutive syndrome in schizophrenia. Psychol Med 27 : 635-646 (1997)

21) Moriyama Y, Mimura M, Kato M, Yoshino A, Hara T, Kashima H, Kato A and Watanabe A : Executive dysfunction and clinical outcome in chronic alcoholics. Alcohol Clin Exp Res 26 : 1239-1244 (2002)

22) Sumiyoshi A : Clinical evaluation of perospirone in outpatients: comparison with risperidone. $J p n ~ J$ Psychopharmacol 6 : 895-904 (2003) 
23) Montes JM, Ciudad A, Gascon J and Gomez JC; EFESO Study Group : Safety, effectiveness, and quality of life of olanzapine in first-episode schizophrenia: a naturalistic study. Prog Neuropsychopharmacol Biol Psychiatry 27 : 667-674 (2003)

24) Weiss EM, Bilder RM and Fleischhacker WW : The effects of second-generation antipsychotics on cognitive functioning and psychosocial outcome in schizophrenia. Psychopharmacology 162:11-17 (2002)

25) Basso MR, Nasrallah HA, Olson SC and Bornstein RA: Neuropsychological correlates of negative, disorganized and psychotic symptoms in schizophrenia. Schizophr Res 31 : 99-111 (1998)

26) Murasaki M : Preclinical and clinical features of perospirone. Jpn J Clin Psychopharmacol 4 : $849-868$ (2001)

27) Rasmusson AM, Goldstein LE, Deutch AY, Bunney BS and Roth RH: 5-HT 1 A agonist \pm 8-OH-DPAT modulates basal and stress-induced changes in medical prefrontal cortical dopamine. Synapse 18: 218-224 (1994)

28) Rollema H, Lu Y, Schimidt AW, Sprouse JS and Zorn SH : 5-HT 1 A receptor activation contributes to ziprasidone-induced dopamine release in rat prefrontal cortex. Biol Psychiatry 48 : 229-237 (2000)

29) Sumiyoshi T, Matsui M, Yamashita I, Nohara S, Uehara T, Kurachi M and Meltzer HY : Effect of adjunctive treatment with serotonin-1A agonist tandospirone on memory functions in schizophrenia. $J$ Clin Psychopharmacol $20: 386-388$ (2000)

30) Kudo $\mathrm{T}$ and Takeda $\mathrm{M}$ : Acute effect of atypical antipsychotics. Jpn J Clin Psychopharmacol 5: 155-165 (2002)

[Received January 28, 2004 : Accepted February 13, 2004] 